\title{
Introduction of a New Score to Assess Surgical Efficiency in Holmium Laser Enucleation of the Prostate
}

\author{
Bernd Rosenhammer Maximilian Schönhärl Roman Mayr Marco J. Schnabel \\ Maximilian Burger Christian Eichelberg \\ Department of Urology, Caritas St. Josef Medical Center, University of Regensburg, Regensburg, Germany
}

\section{Keywords}

Holmium laser enucleation of the prostate $\cdot$ Surgical

experience · Enucleation speed · Laser energy

\begin{abstract}
Introduction: While holmium laser enucleation of the prostate (HoLEP) is accepted as safe and efficient, a long learning curve is considered the main reason for its slow adoption in clinical practice. So far, no standardized and easy-to-use parameter has been implemented to measure surgical experience or efficiency which could be useful for training and quality control purposes. The aim of the present study was to evaluate the learning curves of 2 HoLEP beginners and to identify applicable efficiency outcome measures as well as potentially complicating factors in performing HoLEP. Patients and Methods: A total of 594 patients treated by HoLEP between September 2016 and May 2019 were enrolled. The procedures were initially performed by 1 HoLEP expert (reference surgeon); over time, 2 further surgeons were trained. Baseline characteristics, enucleation weight, morcellation and enucleation time, laser energy usage, and postoperative results were recorded prospectively. The learning curves of the 2 novices were analyzed and compared to the reference surgeon. Logistic regression analyses were performed to identify predictors for postoperative grade $\geq 2$ complica-
\end{abstract}

tions. Results: Median enucleation ratio and complication rates did not significantly alter along the learning curves. Median enucleation speed and laser energy application of the 2 novices significantly improved with growing experience. Combining these variables, we introduced the "HoLEP efficiency score" (HES) which demonstrated the most appropriate value to reflect the surgical experience and efficiency. The median HES for the reference surgeon was $82.8 \mathrm{~min} \mathrm{~kJ} / \mathrm{g}$. For the 2 novices, a drop from 130 and $124.4 \mathrm{~min} \mathrm{~kJ} / \mathrm{g}$ by -57 and $-30 \%$, respectively, was observed. Among several tested clinical parameters, the presence of prostate cancer ( $p=$ 0.047 ) and the surgical caseload $(p<0.001)$ influenced the HES. On multivariable logistic regression, American Society of Anesthesiologists score and prostate cancer were independent predictors for grade $\geq 2$ complications ( $p=0.002$, odds ratio [OR] 2.042 and $p=0.038$, OR 1.940). Conclusion: We introduce the HES as an objective and measurable tool to quantify surgical efficiency. In clinical practice, the HES may find application in training and quality control purposes as well as in comparing surgical modifications and hardware. Patients with prostate cancer seem to be more challenging cases and have a higher risk for complications, and may preferably be treated by experienced surgeons.

C) 2020 The Author(s)

Published by S. Karger AG, Basel karger@karger.com www.karger.com/uin

Karger $\frac{1}{6}$

GOPEN ACCESS
(C) 2020 The Author(s)

Published by S. Karger AG, Basel

This is an Open Access article licensed under the Creative Commons Attribution-NonCommercial-4.0 International License (CC BY-NC) (http://www.karger.com/Services/OpenAccessLicense), applicable to the online version of the article only. Usage and distribution for commercial purposes requires written permission.
Bernd Rosenhammer

Department of Urology

Caritas St. Josef Medical Center, University of Regensburg

Landshuter St. 65, DE-93053 Regensburg (Germany)

bernd.rosenhammer@ukr.de 


\section{Introduction}

In the recent years, holmium laser enucleation of the prostate (HoLEP) has become a main surgical option for treatment of lower urinary tract symptoms due to benign prostatic enlargement when surgical therapy is indicated [1]. In contrast to transurethral resection of the prostate (TURP) and open simple prostatectomy, it can be performed size independently and with lower morbidity [2, 3]. Moreover, apart from similar functional long-term outcomes [4], our group reported a higher prostate cancer detection rate by HoLEP than TURP [5].

HoLEP is judged to have a long learning curve, which may have restricted its widespread use in everyday practice, especially in non-reference centers $[6,7]$. In the past years, several studies have been published investigating the learnability of HoLEP, and a recent review estimated 50 procedures to achieve a stable outcome, which could decrease to approximately 25 in case of a structured training. In these studies, various endpoints were applied to describe the learning curve, such as enucleation and morcellation efficiency, postoperative complications, functional outcome, postoperative prostate-specific antigen (PSA) decrease, lack of conversion to TURP, and laser energy application [8-23]. Further parameters may influence the learning curve, including patient-related factors potentially complicating the procedure. With HoLEP's excellent outcomes and its increasing status in disobstructive surgery, a structured and widespread implementation in the urological community is desirable. In this context, objective parameters or even 1 single score describing the surgical experience or efficiency could be helpful in several aspects: standardization of HoLEP education, case selection for novices, reduction of complications, quality control, comparison of new laser devices, or modifications within the procedure.

The following study was conducted to report real-life experiences from a German university single-center during and after the implementation of HoLEP. The purpose was to identify appropriate outcome measures reflecting the surgical experience and efficiency in performing HoLEP. Moreover, we aimed to evaluate potentially complicating factors in order to ease patient selection for HoLEP beginners.

\section{Patients and Methods}

\section{Surgical Setting}

Since the introduction of HoLEP in our department in September 2016, a total of 606 patients were treated until May 2019, which was the data cutoff point for the present study. All procedures were performed using a Lumenis ${ }^{\circledR}$ laser generator and a Richard Wolf ${ }^{\circledR}$ Piranha ${ }^{\mathrm{TM}}$ morcellator.

Initially, only 1 surgeon (surgeon $1-\mathrm{CE}$ ), who was already a HoLEP expert, was designated to perform the procedure. This surgeon was stated as the reference surgeon for the data analyses. Starting in August 2017 and March 2018, respectively, 2 further surgeons (RM and MJS) were trained by a stepwise approach under the supervision of surgeon 1 . Notably, both surgeons were already experienced TURP surgeons.

\section{Study Design and Data Collection}

The study protocol was approved by the Ethics Committee of the University of Regensburg. All patients were consecutively added to a prospectively held database. The preoperative workup included the following parameters: patient age, American Society of Anesthesiologists (ASA) score, serum PSA level, prostate volume measured by transrectal ultrasound, anticoagulation/antiplatelet therapy, indwelling catheter (none/transurethral/suprapubic), preoperative urinary tract infection (UTI), history of any previous prostate biopsy, recent prostate biopsy ( $<8$ weeks), and known prostate cancer. Antiplatelet drugs were continued in all cases. Bridging with low molecular weight heparin was performed in patients on oral vitamin $\mathrm{K}$ antagonists, whereas anticoagulation with dabigatran/rivaroxaban/apixaban/edoxaban was paused $48 \mathrm{~h}$ before and at least 7 days after the procedure depending on the presence of gross hematuria (low molecular weight heparin bridging/switching was used). Patients with UTIs were treated based on urine culture results, starting at least $24 \mathrm{~h}$ before the procedure.

The perioperative data collection included enucleated prostate weight (g), enucleation ratio defined as percentage (\%) of retrieved tissue in relation to preoperative prostate transrectal ultrasound volume, enucleation and morcellation time, enucleation and morcellation speed defined as time/enucleated weight $(\mathrm{min} / \mathrm{g})$, total energy delivered $(\mathrm{kJ})$, and energy/enucleated weight $(\mathrm{kJ} / \mathrm{g})$. Postoperative complications within 90 days were investigated retrospectively and stratified according to the Clavien-Dindo (CD) classification [24]. Moreover, the histopathological results of the removed specimens were recorded. Due to insufficient documentation of intraoperative data, 12 patients had to be excluded, allowing for a total of 594 patients to be analyzed.

\section{Statistical Analysis}

For all statistical analyses, SPSS version 24.0 (Chicago, IL, USA) was used. Continuous variables were presented as mean (standard deviation, SD) or median (interquartile range, IQR), and categorical variables as absolute numbers and percentages. $\chi^{2}$ test and Fisher's exact test were used to compare categorical variables. ANOVA and the Kruskal-Wallis test were used to compare continuous baseline variables between the 3 surgeons depending on normal or non-normal distribution. The MannWhitney $\mathrm{U}$ test was applied to compare continuous outcome variables of surgeons 2 and 3 , and surgeon 1 . Normal distribution was tested by the Shapiro-Wilk test. Logistic regression analyses were performed to identify potential predictors for postoperative complications. $p$ values $<0.05$ were considered statistically significant. 
Table 1. Patient characteristics

\begin{tabular}{|c|c|c|c|c|c|}
\hline Patients, $\mathrm{n}$ & 594 & 181 & 275 & 138 & - \\
\hline Patient age, mean (SD), years & $70.9(8.4)$ & $71.2(8.2)$ & $71.1(8.4)$ & $69.8(8.7)$ & 0.204 \\
\hline \multicolumn{6}{|l|}{ ASA score, $n(\%)$} \\
\hline I & $41(6.9)$ & $17(9.4)$ & $15(5.5)$ & $9(6.5)$ & \multirow[t]{3}{*}{0.411} \\
\hline II & $333(56.1)$ & $98(54.1)$ & $157(57.1)$ & $78(56.5)$ & \\
\hline III & $212(35.7)$ & $64(35.4)$ & $97(35.3)$ & $51(37.0)$ & \\
\hline \multicolumn{6}{|l|}{ Preoperative indwelling catheter, $n(\%)$} \\
\hline None & $389(65.5)$ & $117(64.6)$ & $180(65.5)$ & $92(66.7)$ & \multirow[t]{3}{*}{0.825} \\
\hline Transurethral & $169(28.5)$ & $50(27.6)$ & $81(29.5)$ & $38(27.5)$ & \\
\hline Suprapubic & $36(6.0)$ & $14(7.7)$ & $14(5.1)$ & $8(5.8)$ & \\
\hline \multicolumn{6}{|l|}{ Preoperative UTI, $n(\%)$} \\
\hline Yes & $202(34.0)$ & $71(39.2)$ & $94(34.2)$ & $37(26.8)$ & 0.068 \\
\hline Preoperative prostate volume, median (IQR), cc & $85.0(44.0)$ & $93.0(43.0)$ & $80.0(45.0)$ & $75.0(40.0)$ & $<0.001$ \\
\hline Preoperative total PSA, median (IQR), ng/mL & $4.86(5.84)$ & $5.31(5.50)$ & $4.73(5.62)$ & $4.22(6.80)$ & 0.212 \\
\hline \multicolumn{6}{|l|}{ Any previous prostate biopsy, $n(\%)$} \\
\hline Yes & $164(27.6)$ & $47(26.0)$ & $75(27.3)$ & $42(30.4)$ & \multirow[t]{2}{*}{0.667} \\
\hline No & $430(72.4)$ & $134(74.0)$ & $200(72.7)$ & $96(69.6)$ & \\
\hline \multicolumn{6}{|l|}{ Prostate biopsy within 8 weeks before HoLEP, $n(\%)$} \\
\hline Yes & $61(10.3)$ & $19(10.5)$ & $28(10.2)$ & $14(10.1)$ & \multirow[t]{2}{*}{0.993} \\
\hline No & $533(89.7)$ & $162(89.5)$ & $247(89.8)$ & $124(89.9)$ & \\
\hline \multicolumn{6}{|l|}{ Preoperative known prostate cancer, $n(\%)$} \\
\hline Yes & $25(4.2)$ & $4(2.2)$ & $6(2.2)$ & $15(10.9)$ & \multirow[t]{2}{*}{$<0.001$} \\
\hline No & $569(95.8)$ & $177(97.8)$ & $269(97.8)$ & $123(89.1)$ & \\
\hline
\end{tabular}

Bold values indicate $p$ values $<0.05$. UTI, urinary tract infection; Tx, therapy; PSA, prostate-specific antigen; HoLEP, holmium laser enucleation of the prostate; ASA, American Society of Anesthesiologists.

\section{Results}

\section{Baseline Characteristics}

A total of 594 patients were included into the data evaluation; 181 procedures were performed by surgeon 1 (September 2016-December 2017), 275 by surgeon 2 (August 2017-May 2019), and 138 by surgeon 3 (March 2018-May 2019). The patients' characteristics for the entire cohort and the subgroups operated on by each of the 3 surgeons are shown in Table 1. The baseline parameters were balanced regarding age, ASA score, indwelling catheter, UTIs, anticoagulation or antiplatelet aggregation therapies, serum PSA, and previous prostate biopsies. None of the patients had a history of prostate radiotherapy. A significant difference in preoperative prostate volume was observed, with a median volume of 93/80/75 cc for surgeon $1 / 2 / 3$, respectively $(p<0.001)$. Another imbalance was noticed with regard to patients with known prostate cancer. Whereas the preoperatively diagnosed cancer rate for surgeons 1 and 2 was $2.2 \%$, a higher rate of $10.9 \%$ was noted for surgeon $3(p<0.001)$.

\section{Operative Results}

Although the median retrieved specimen weight differed among the 3 surgeons with 62/56/51 $\mathrm{g}$ for surgeon $1 / 2 / 3$ (compared to surgeon $1: p=0.011$ and $p<0.001$ for surgeons 2 and 3 , respectively), the enucleation ratio was comparable as shown in Table 2a. This was consistent with the described differences in prostate baseline volumes. The rate of newly diagnosed prostate cancer in the histopathological specimen was $10.9 \%$, with a comparable distribution among the 3 surgeons (12.2/9.9/11.6\% for surgeon $1 / 2 / 3$ ).

The median morcellation times for surgeon $1 / 2 / 3$ were $12 / 10 / 8 \min (p=0.004$ and $p<0.001)$, respectively. Regarding morcellation speed, the differences were margin- 
Table 2. Operative results describing the surgical experience and analysis of HES



All statistical analyses performed in comparison to surgeon 1 (reference). Bold values indicate $p$ values $<0.05$. HES, HoLEP efficiency score; HoLEP, holmium laser enucleation of the prostate. 


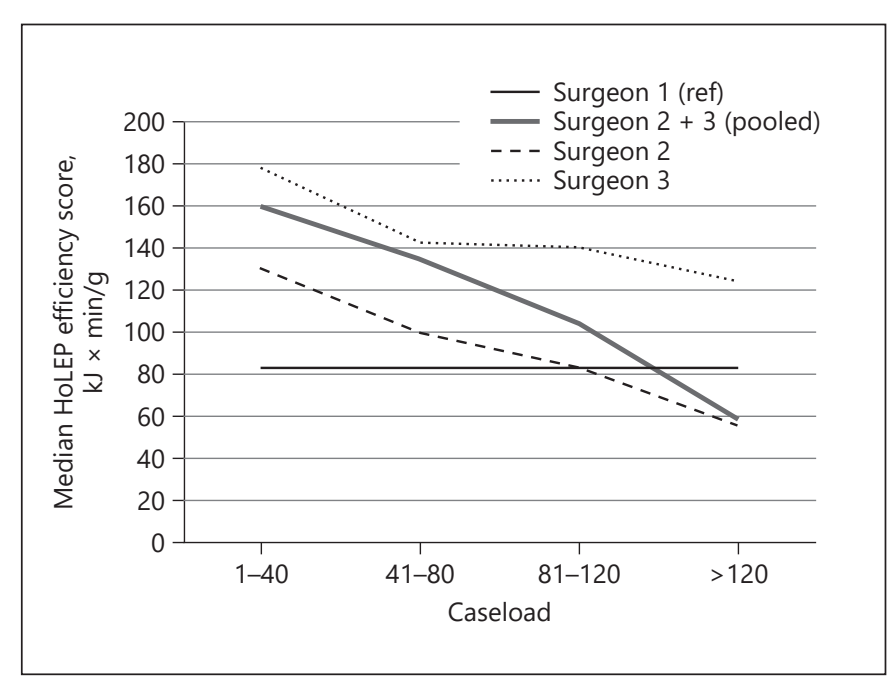

Fig. 1. Development of the HES with increasing caseload. HoLEP, holmium laser enucleation of the prostate; HES, HoLEP efficiency score. Surgeon 1 = reference.

al (median 0.19/0.18/0.15 min/g, $p=0.066$ and $p<0.001$ ). The results for enucleation time, enucleation speed, laser energy usage, and laser energy usage per gram tissue weight are shown in detail in Table $2 \mathrm{a}$ and revealed significant differences for surgeons 2 and 3 compared to surgeon 1.

The overall complication rate was 22.1 , with $10.4 \%$ being $\mathrm{CD}$ grade $\geq 2$. There were no significant differences between the 3 surgeons. Apart from 1 patient requiring blood transfusion, all CD grade 2 complications were infectious. CD grade 3 complications mainly included coagulation or transurethral resection of remaining prostatic tissue or blood clots causing obstruction.

\section{Introduction of the "HoLEP Efficiency Score"}

Based on the previous results, we stratified the parameters enucleation ratio, enucleation time, enucleation speed, laser energy, and complication rates by the number of procedures performed by surgeons 2 and 3 in order to assess potential development with growing experience (Table $2 \mathrm{a}$ ). The following intervals were applied: 1-40, $41-80,81-120$, and $>120$ performed HoLEP procedures; the results of surgeon 1 were the statistical reference for all tests.

The enucleation ratio of $60-65 \%$ of preoperative prostate volume did not significantly change with higher caseload. Similarly, no trend in postoperative complication rates was noted. In contrast, the enucleation time and speed significantly decreased for both sur-
Table 3. Evaluation of factors potentially influencing the HES

\begin{tabular}{|c|c|c|c|}
\hline Variable & $N$ & $\begin{array}{l}\text { Median HES, } \\
\operatorname{min~} \mathrm{kJ} / \mathrm{g}\end{array}$ & $p$ value \\
\hline \multicolumn{4}{|c|}{ Preoperative indwelling transurethral catheter } \\
\hline Yes & 169 & 92.3 & \multirow[t]{2}{*}{0.911} \\
\hline No & 425 & 85.7 & \\
\hline \multicolumn{4}{|c|}{ Preoperative UTI } \\
\hline Yes & 202 & 83.7 & \multirow[t]{2}{*}{0.238} \\
\hline No & 392 & 93.1 & \\
\hline \multicolumn{4}{|c|}{ Anticoagulation/antiplatelet therapy } \\
\hline Yes & 222 & 84.6 & \multirow[t]{2}{*}{0.235} \\
\hline No & 372 & 92.7 & \\
\hline \multicolumn{4}{|c|}{ Prostate biopsy within 8 weeks before HoLEP } \\
\hline Yes & 61 & 90.0 & \multirow[t]{2}{*}{0.505} \\
\hline No & 533 & 90.5 & \\
\hline \multicolumn{4}{|c|}{ Prostate cancer (known or incidental) } \\
\hline Yes & 90 & 117.3 & \multirow[t]{2}{*}{0.047} \\
\hline No & 504 & 89.4 & \\
\hline \multicolumn{4}{|c|}{ Caseload (no. of procedures performed, cases pooled) } \\
\hline $1-40$ & 80 & 160.0 & \multirow[t]{4}{*}{$<\mathbf{0 . 0 0 1}$} \\
\hline $40-80$ & 80 & 134.5 & \\
\hline $80-120$ & 80 & 104.2 & \\
\hline$>120$ & 354 & 74.3 & \\
\hline
\end{tabular}

Bold values indicate $p$ values $<0.05$. HoLEP, holmium laser enucleation of the prostate; UTI, urinary tract infection; HES, HoLEP efficiency score.

geons 2 and 3 through their learning curve. The enucleation speed of surgeon 2 declined from $0.98 \mathrm{~min} / \mathrm{g}$ by $-41 \%$ and the speed of surgeon 3 from $1.16 \mathrm{~min} / \mathrm{g}$ by $-16 \%$.

Analogous to the development of the enucleation speed, the median laser energy usage continuously decreased from $147.5 \mathrm{~kJ}$ by $-36 \%$ for surgeon 2 and from $150.0 \mathrm{~kJ}$ by $-23 \%$ for surgeon 3 (see Table $2 \mathrm{a}$ ). As enucleation speed and applied laser energy demonstrated the most distinct change during the learning curves, we decided to combine enucleation time, laser energy, and retrieved tissue weight to a single score - the HoLEP efficiency score (HES; min $\mathrm{kJ} / \mathrm{g}$ ). When stratifying the HES by caseload again, we observed a clear correlation with the surgical experience as the median score consistently decreased (Table 2b; Fig. 1).

In a next step, we tested for factors that might complicate or influence a HoLEP procedure and thereby potentially alter the HES: preoperative presence of transurethral catheter, preoperative UTI, anticoagulation/antiaggregation therapy, preoperative history of prostate biopsy within 8 weeks, presence of prostate cancer (known or 
Table 4. Potential predictors for CD grade $\geq 2$ complications, logistic regression analyses

\begin{tabular}{|c|c|c|c|c|}
\hline \multirow[t]{2}{*}{ Variable } & \multicolumn{2}{|c|}{ Univariate } & \multicolumn{2}{|c|}{ Multivariate } \\
\hline & $p$ value & OR $(95 \% \mathrm{CI})$ & $p$ value & OR $(95 \% \mathrm{CI})$ \\
\hline Age & 0.137 & $1.025(0.992-1.058)$ & - & - \\
\hline ASA score & 0.001 & $2.112(1.359-3.281)$ & 0.002 & $2.042(1.306-3.195)$ \\
\hline $\mathrm{P}-\mathrm{Bx}<8$ weeks before HoLEP & 0.780 & $1.127(0.489-2.597)$ & - & - \\
\hline Anticoagulation/antiplatelet $\mathrm{Tx}$ & 0.060 & $1.660(0.979-2.816)$ & - & - \\
\hline Transurethral catheter & 0.030 & $1.821(1.059-3.131)$ & - & - \\
\hline UTI & 0.096 & $1.574(0.923-2.683)$ & - & - \\
\hline Prostate volume & 0.903 & $1.000(0.994-1.007)$ & - & - \\
\hline Prostate cancer & 0.015 & $2.153(1.159-4.000)$ & 0.038 & $1.940(1.036-3.633)$ \\
\hline \multicolumn{5}{|l|}{ Surgeon (Ref. surgeon 1) } \\
\hline Surgeon 2 & 0.315 & $0.729(0.393-1.352)$ & - & - \\
\hline Surgeon 3 & 0.845 & $1.070(0.541-2.116)$ & - & - \\
\hline \multicolumn{5}{|l|}{ Caseload (ref. >120) } \\
\hline $0-40$ & 0.443 & $1.326(0.645-2.723)$ & - & - \\
\hline $40-80$ & 0.231 & $0.554(0.211-1.456)$ & - & - \\
\hline $80-120$ & 0.847 & $0.924(0.413-2.065$ & - & - \\
\hline HES & 0.872 & $1.000(0.997-1.003)$ & & \\
\hline
\end{tabular}

Bold values indicate $p$ values $<0.05$. CD, Clavien-Dindo; $\mathrm{P}-\mathrm{Bx}$, prostate biopsy; UTI, urinary tract infection; Tx, therapy; Ref., reference; OR, odds ratio; 95\% CI, 95\% confidence interval; HES, HoLEP efficiency score; HoLEP, holmium laser enucleation of the prostate; ASA, American Society of Anesthesiologists.

incidental), and overall surgical experience. For the latter, we stepwise pooled cases of surgeons 2 and 3 from each subgroup of 40 patients and additionally assigned all cases by reference surgeon 1 to the last subgroup " $>120$ cases." The results are shown in Table 3. Among the patientrelated parameters, only the presence of prostate cancer (known or incidental) had a significant influence and resulted in a $>30 \%$ rise of median HES ( $p=0.047$ ). Contrarily, the median score significantly decreased by $54 \%$ with a higher surgical caseload $(p<0.001)$.

\section{Risk Factors for Postoperative Complications}

To identify potential predictors for the presence of postoperative CD grade $\geq 2$ complications, logistic regression analyses were performed (Table 4). On univariate analysis, ASA score, the presence of prostate cancer, and a preoperative necessity for a transurethral catheter were significantly associated with $C D \geq 2$ complications ( $p=0.001, p=0.030$, and $p=0.015$, respectively). On multivariate analysis, ASA score and prostate cancer were independent predictors for the presence of $\mathrm{CD}$ grade $\geq 2$ complications $(p=0.002$, OR 2.042 and $p=$ 0.038 , OR 1.940). Of note, neither the surgeon nor the caseload nor the HES was associated with CD grade $\geq 2$ complications.

A New Score to Assess HoLEP Efficiency

\section{Discussion}

More than 20 years after the first description of HoLEP, the long learning curve is still considered one of the main reasons for impeding its widespread adoption. Quantification and standardization of the surgical experience could be helpful in several aspects such as a structured training program and case selection for novice HoLEP surgeons. Moreover, even experienced surgeons might benefit from a standardized measurement method, for example, in terms of continuous quality control and reporting as well as for the comparison of new laser devices or modifications within the procedure (e.g., 2-lobe vs. en bloc technique). In the past years, many data have been published on this, providing varying outcome measures to describe the initial learning curve. In the present study, we report on our HoLEP experiences beyond the very initial learning curve. With almost 600 patients and the evaluation of 3 different surgeons, this is one of the largest studies on this topic.

With regard to the overall surgical experience, we mainly focused on the enucleation skills, as we found no relevant differences concerning the morcellation parameters. As suggested previously, the morcellation seems to plateau rather early during the initial learning curve [8]. 
This was confirmed by 3 further studies showing no significant change in morcellation time or efficiency throughout the learning curves, too $[9,10,21]$.

Also in line with previous reports, we observed a significant gain in the enucleation speed along the learning curve, but no relevant alteration of the enucleation ratio [7]. This corroborates HoLEP as an efficient and enduring method in treating prostate hyperplasia, since even novice surgeons might need more time, but will achieve a thorough disobstruction.

Furthermore, laser energy outcome measures have been evaluated in prior studies, and a reduction of laser time and energy usage was observed with growing experience $[11,17,20,22,23]$. Our data underline these findings, showing a continuous improvement for both novice surgeons which was present even beyond case 120 .

Concerning the value of postoperative complication rates in assessing experience, conflicting results have been reported and some groups reported fewer complications with higher caseload $[13,15,20]$. Analogous to other investigators $[21,25]$, we did not observe relevant changes in the here presented study.

We decided to evaluate potential predictors for complications limited to the more relevant $\mathrm{CD}$ grade $\geq 2$. In line with previous data $[26,27]$, their occurrence was low in our study; in particular, the rate of blood transfusions or repeat surgery was only 5\%. According to our results, complication rates were not dependent on the surgical caseload, which underlines that HoLEP can be safely performed even by beginners. While some potential factors such as anticoagulation/antiplatelet therapy, UTI, or indwelling transurethral catheter had no significant impact, we found that the presence of prostate cancer was an independent predictor for postoperative $\mathrm{CD}$ grade $\geq 2 \mathrm{com}$ plications.

Based on our results for enucleation speed and laser energy usage, we introduced the HES to quantify the surgical experience. For both HoLEP novices, a distinctive drop of 58\% (surgeon 2) and 30\% (surgeon 3) was observed along the learning curve. According to these findings, the HES seems to be a valuable tool to reflect the surgical experience and enables an objective efficiency quantification.

A similar combination of these parameters has recently been introduced in a study by Kim et al. [23], who also concluded that this is a more sensitive tool for evaluating enucleation skills. Their report was based on 100 consecutive patients and thereby roughly $1 / 6$ th of the here reported number of cases. Furthermore, the study was limited to the investigation of a single surgeon's learning curve, while the strength of our study is the evaluation of 2 HoLEP novices compared to an experienced reference surgeon. In contrast to our results, the cited study observed a plateau at 61-70 cases, whereas in our study no plateau was reached. Aside from the single-surgeon nature of the study, one further explanation might be found in differing patient characteristics.

Their median baseline prostate volume was only $53 \mathrm{cc}$ compared to $85 \mathrm{cc}$ in the present study. Consequently, the median resection weight, enucleation time, and consumed energy were higher in our study. Moreover, prostate cancer rates were not reported in detail, while we could demonstrate a significant impact on the HES by this patient-related factor. In these cases, we found a significantly higher score, which seems to characterize these procedures as more complex with longer duration and/or higher laser energy usage. Combined with the fact that prostate cancer was also a significant predictor for postoperative complications, we would cautiously recommend that patients with known or expected prostate cancer should preferably be treated by experienced surgeons rather than by HoLEP beginners.

Our study has some limitations: While baseline parameters and the intraoperative outcome measures were recorded prospectively, the evaluation of postoperative complications was performed in a retrospective manner. Hereby, particularly grade 1 complications possibly might have been overlooked. To avoid this bias, we limited our regression analyses to grade $\geq 2$ complications. Apart from a more reliable documentation, these higher grade complications are more relevant in clinical practice anyway. Moreover, we are not able to provide data concerning the functional results and potential differences among the surgeons and along the learning curve.

When HoLEP was implemented in our department, it was used instead of open prostatectomy, preferably for larger glands. With growing experience and decent functional results, we also applied HoLEP to smaller glands. This corresponds to the observed differences in baseline volumes and enucleation weights among the 3 surgeons, with the lowest numbers for the latest trained surgeon 3.

Despite having evaluated 2 HoLEP novices compared to a third reference surgeon, our results certainly need validation by the investigation of larger collectives and more surgeons, preferably in a multicenter setting. Nevertheless, we think that the HES might have the potential to standardize surgical experience and efficiency in performing HoLEP. This could be helpful for training and quality control purposes, as well as to compare modifications of the procedure itself - for example, 2-lobe versus 
en bloc technique - or quantifying potential benefits by new endoscopic or laser devices and their features, such as the introduction of laser coagulation modes. In our department, for instance, we started using the HES regularly to monitor the training of all novel HoLEP surgeons.

\section{Conclusion}

In the present study, we introduce the HES as a combination of enucleation efficiency and laser energy usage. We proved a distinctive decline of this score with increasing surgical caseload, providing an objective tool to quantify surgical efficiency. For clinical practice, we propose that the HES could be applied for training and quality control purposes as well as for comparing surgical or hardware modifications. Patients with known or expected prostate cancer seem to be more complex cases with higher risk for complications and may be preferably treated by more experienced surgeons than by HoLEP beginners.

\section{Statement of Ethics}

The study was performed following the approval of the local research ethics committee (reference number 17-777-101).

\section{Conflict of Interest Statement}

The authors declare to have no conflicts of interest.

\section{Funding Sources}

There was no funding for this study.

\section{Author Contributions}

Study concept and design: Rosenhammer and Eichelberg. Acquisition of data: Rosenhammer, Schönhärl, Mayr, Schnabel, and Eichelberg. Analysis and interpretation of data: Rosenhammer, Schönhärl, and Eichelberg. Drafting of the manuscript: Rosenhammer and Eichelberg. Critical revision of the manuscript for important intellectual content: Mayr and Schnabel, Burger. Statistical analysis: Rosenhammer and Schönhärl. Supervision: Burger and Eichelberg.

\section{References}

1 Gravas S, Bach T, Bachmann A, Drake M, Gacci M, Gratzke C, et al. https://uroweb.org/ wp-content/uploads/EAU-Guidelines-onNon-Neurogenic-Male-LUTS-incl.BPO-2020.pdf

2 Kuntz RM, Lehrich K, Ahyai S. Does perioperative outcome of transurethral holmium laser enucleation of the prostate depend on prostate size? J Endourol. 2004;18(2) 183-8.

3 Gazel E, Kaya E, Yalcin S, Aybal HÇ, Aydogan TB, Tunc L. Comparison of the efficacy of holmium laser enucleation of the prostate in treating prostate volumes of $\leq 80$ and $>80 \mathrm{~mL}$. Urol Int. 2019;102(3):306-10.

4 Cornu JN, Ahyai S, Bachmann A, de la Rosette J, Gilling P, Gratzke C, et al. A systematic review and meta-analysis of functional outcomes and complications following transurethral procedures for lower urinary tract symptoms resulting from benign prostatic obstruction: an update. Eur Urol. 2015;67(6): 1066-96.

5 Rosenhammer B, Lausenmeyer EM, Mayr R, Burger M, Eichelberg C. HoLEP provides a higher prostate cancer detection rate compared to bipolar TURP: a matched-pair analysis. World J Urol. 2018;36(12):203541.

A New Score to Assess HoLEP Efficiency
6 Gravas S, Bachmann A, Reich O, Roehrborn CG, Gilling PJ, De La Rosette J. Critical review of lasers in benign prostatic hyperplasia (BPH). BJU Int. 2011;107(7): 1030-43.

7 Kampantais S, Dimopoulos P, Tasleem A, Acher P, Gordon K, Young A. Assessing the learning curve of holmium laser enucleation of prostate (HoLEP). A systematic review. Urology. 2018;120:9-22.

8 El-Hakim A, Elhilali MM. Holmium laser enucleation of the prostate can be taught: the first learning experience. BJU Int. 2002;90(9): 863-9.

9 Seki N, Mochida O, Kinukawa N, Sagiyama K, Naito S. Holmium laser enucleation for prostatic adenoma: analysis of learning curve over the course of 70 consecutive cases. J Urol. 2003;170(5):1847-50.

10 Shah HN, Mahajan AP, Sodha HS, Hegde S, Mohile PD, Bansal MB. Prospective evaluation of the learning curve for holmium laser enucleation of the prostate. J Urol. 2007; 177(4):1468-74.

11 Elzayat EA, Elhilali MM. Holmium laser enucleation of the prostate (HoLEP): long-term results, reoperation rate, and possible impact of the learning curve. Eur Urol. 2007;52(5): 1465-71.
12 Bae J, Oh SJ, Paick JS. The learning curve for holmium laser enucleation of the prostate: a single-center experience. Korean J Urol. 2010; 51(10. 688-93.

13 Jeong CW, Oh JK, Cho MC, Bae JB, Oh SJ. Enucleation ratio efficacy might be a better predictor to assess learning curve of holmium laser enucleation of the prostate. Int Braz J Urol. 2012;38(3):362-72;

14 Robert G, Cornu JN, Fourmarier M, Saussine C, Descazeaud A, Azzouzi AR, et al. Multicentre prospective evaluation of the learning curve of holmium laser enucleation of the prostate (HoLEP). BJU Int. 2016;117(3):4959.

15 Brunckhorst $\mathrm{O}$, Ahmed $\mathrm{K}$, Nehikhare $\mathrm{O}$, Marra G, Challacombe B, Popert R. Evaluation of the learning curve for holmium laser enucleation of the prostate using multiple outcome measures. Urology. 2015;86(4):824-

16 Peyronnet B, Robert G, Comat V, Rouprêt M, Gomez-Sancha F, Cornu JN, et al. Learning curves and perioperative outcomes after endoscopic enucleation of the prostate: a comparison between GreenLight 532-nm and holmium lasers. World J Urol. 2017;35(6):97383. 
17 Placer J, Gelabert-Mas A, Vallmanya F, Manresa JM, Menéndez V, Cortadellas $\mathrm{R}$, et al. Holmium laser enucleation of prostate: outcome and complications of self-taught learning curve. Urology. 2009;73(5):1042-8.

18 Du C, Jin X, Bai F, Qiu Y. Holmium laser enucleation of the prostate: the safety, efficacy, and learning experience in China. J Endourol. 2008;22(5): 1031-6.

19 Hwang JC, Park SM, Lee JB. Holmium laser enucleation of the prostate for benign prostatic hyperplasia: effectiveness, safety, and overcoming of the learning curve. Korean J Urol. 2010;51(9):619-24.

20 Elshal AM, Nabeeh H, Eldemerdash Y, Mekkawy R, Laymon M, El-Assmy A, et al. Prospective assessment of learning curve of holmium laser enucleation of the prostate for treatment of benign prostatic hyperplasia using a multidimensional approach. J Urol. 2017;197(4):1099-107.
21 Shigemura K, Yamamichi F, Kitagawa K, Yamashita M, Oka Y, Tanaka H, et al. Does surgeon experience affect operative time, adverse events and continence outcomes in holmium laser enucleation of the prostate? A review of more than 1,000 cases. J Urol. 2017;198(3):663-70.

22 Dusing MW, Krambeck AE, Terry C, Matlaga BR, Miller NL, Humphreys MR, et al. Holmium laser enucleation of the prostate: efficiency gained by experience and operative technique. J Urol. 2010;184(2):635-40.

$23 \mathrm{Kim} \mathrm{KH,} \mathrm{Kim} \mathrm{KT,} \mathrm{Oh} \mathrm{JK,} \mathrm{Chung} \mathrm{KJ,} \mathrm{Yoon} \mathrm{SJ,}$ Jung $\mathrm{H}$, et al. Enucleated weight/enucleation time, is it appropriate for estimating enucleation skills for holmium laser enucleation of the prostate? A consideration of energy consumption. World J Mens Health. 2018;36(1):79-86.

24 Dindo D, Demartines N, Clavien PA. Classification of surgical complications: a new proposal with evaluation in a cohort of $6336 \mathrm{pa}$ tients and results of a survey. Ann Surg. 2004; 240(2):205-13.
25 Soto-Mesa D, Amorín-Díaz M, Pérez-Arviza L, Fernández-Pello Montes S, Martín-Huéscar A. Holmium laser enucleation of the prostate and retropubic prostatic adenomectomy: morbidity analysis and anesthesia considerations. Actas Urol Esp. 2015;39(9):535-45.

26 Ahyai SA, Gilling P, Kaplan SA, Kuntz RM, Madersbacher S, Montorsi F, et al. Meta-analysis of functional outcomes and complications following transurethral procedures for lower urinary tract symptoms resulting from benign prostatic enlargement. Eur Urol. 2010; 58(3):384-97.

27 Shah HN, Mahajan AP, Hegde SS, Bansal MB Peri-operative complications of holmium laser enucleation of the prostate: experience in the first 280 patients, and a review of literature. BJU Int. 2007;100(1):94-101. 